\title{
Hydrodynamic stress on fractal aggregates of spheres
}

\author{
G. Bossis and A. Meunier
}

Laboratoire de Physique de la Matière Condensée, Université de Nice-Sophia Antipolis, Parc Valrose, 06034

Nice Cédex, France

J. F. Brady

Department of Chemical Engineering, California Institute of Technology, Pasadena, California 91125

(Received 23 April 1990; accepted 4 December 1990)

We calculate the average hydrodynamic stress on fractal aggregates of spheres using Stokesian dynamics. We find that for fractal aggregates of force-free particles, the stress does not grow as the cube of the radius of gyration, but rather as the number of particles in the aggregate. This behavior is only found for random aggregates of force-free particles held together by hydrodynamic lubrication forces. The stress on aggregates of particles rigidly connected by interparticle forces grows as the radius of gyration cubed. We explain this behavior by examining the transmission of the tension along connecting lines in an aggregate and use the concept of a persistance length in order to characterize this stress transmission within an aggregate.

\section{INTRODUCTION}

The hydrodynamic properties of fractal aggregates have been studied primarily through the relationship between their hydrodynamic radius and their gyration radius. Some authors have modeled the fractal aggregates as a porous medium governed by Brinkman's equation ${ }^{1,2}$ with a force density proportional to the local number density of particles, which decreases with distance $r$ from the center of the aggregate as $r^{d_{t}-3}$, where $d_{f}$ is the fractal dimension.

The case we are studying is rather different, however, since we do not consider rigid links between the particles of the aggregate. The spheres are in close contact, but are still able to rotate and translate relative to one another. This internal motion is resisted by hydrodynamic lubrication forces and, although the relative motion is very small, it nevertheless has a profound effect on the stress transmission. In this case of "free" aggregates (i.e., force- and torque-free particles), the hydrodynamic stress will secm to grow as the number $N$ of particles making up the aggregate. This is to be contrasted with the case of "fixed" aggregates where the particles are rigidly connected by interparticle forces-where the hydrodynamic stress grows as the cube of the largest dimension, e.g., as the cube of the radius of gyration. Thus, the free vs fixed behavior represent opposite extremes in the possible behavior of aggregates of particles and it will be shown below that this is indeed the case.

Colloidal particles may aggregatc in such a way as to form either fixed or free structure depending on the nature of the aggregation processes and the type of interparticle bonds established. In many kinetically driven aggregation processes, the particles fall into the deep London-van der Waals attractive well and are essentially rigidly connected (at least at low amplitude strain rates). In other hydrodynamically driven processes, such as sedimentation, flow through porous media, and shearing motion, however, the aggregated particles are held together only weakly, either by hydrodynamic lubrication forces alone or by the weaker secondary minimum displayed by several forms of interparticle poten- tial. In this case, the aggregates are properly considered free. ${ }^{3.4}$

This profound difference in the scaling behavior of the stress on free and fixed aggregates $N$ vs $N^{3 / d_{f}}$ raises an interesting question regarding the behavior of aggregates which are neither free nor fixed, but rather connected by springlike interparticle forces as in the case of some colloidal aggregates and more commonly in the case of flexible polymers. Which behavior do these display? The answer is both. The high frequency dynamic viscosity of flexible polymers is givm en by the hydrodynamic stress, and as we show below, this scales linearly with the number of particles $N$. At very high frequencies, the springlike connection between polymer segments are note distorted and the polymer appears free. (Free in this context does not imply free draining as employed in the polymer literature.) Indeed, it is just the hydrodynamic interactions between particles neglected in the free draining polymer theories that gives rise to the stress. Thus, we predict that the high frequency dynamic viscosity of a flexible polymer solution should exceed the solvent viscosity by an amount that scales linearly with the molecular weight of the polymer. At low frequencies, however, the polymer is stretched by the shearing motion and the springlike restoring forces act as rigid links, and the behavior will be that of a fixed aggregate with the stress growing as the cube of the radius of gyration, a well-known result.

To illustrate these behaviors, we first recall briefly the model used to calculate the stress by Stokesian dynamics and give the essential relations needed to derive the average stress on an aggregate. In the second part, we present the results obtained for random fractal aggregates. Finally, we discuss these results and the $N$ vs $N^{3 / d_{f}}$ behavior with the help of some special configurations going from the extremes of a straight line to a randomly coiled polymer chain.

\section{THEORY}

We use the formalism already developed to simulate the trajectories of spheres in a Stokes flow. ${ }^{5,6}$ The hydrodynam- 
ic forces and torques $F$ and the stresses $S$ on the particles are related to the velocities $U$ of the particles relative to the external linear flow $U^{\infty}$ by

$$
\left(\begin{array}{l}
F \\
S
\end{array}\right)=-\left(\begin{array}{ll}
R_{F U} & R_{F E} \\
R_{S U} & R_{S E}
\end{array}\right) \cdot\left(\begin{array}{c}
U-U^{\infty} \\
-E^{\infty}
\end{array}\right)
$$

where $E^{\infty}$ is the rate of strain of the imposed linear flow. For $N$ particles, the force and velocity vectors have $6 N$ components ( $3 N$ for translation and $3 N$ for rotation); the symmetric and traceless part of $S=\left(S_{11}, S_{12}, S_{13}, S_{23}, S_{22}\right)$ and $E^{\infty}=\left(E_{11}^{\infty}-E_{33}^{\infty}, 2 E_{12}^{\infty}, 2 E_{13}^{\infty}, 2 E_{23}^{\infty}, E_{22}^{\infty}-E_{33}^{\infty}\right) \quad$ each have $5 N$ components.

In all the equations, the force is normalized by $6 \pi \mu a$, the torque by $6 \pi \mu a^{2}$, and the stress by $20 \pi \mu a^{3} / 3$, where $\mu$ is the viscosity of the solvent and $a$ is the radius of a spherical particle. Here all particles are assumed to be the same size.

The elements of the resistance matrix $R$ are calculated as described previously by adding a two-body contribution $R_{2 b}-R_{2 b}^{\infty}$ coming from lubrication theory for two spheres almost at contact, and a many-body contribution $M^{-1}$, which includes the two first moments of the force distribution on each sphere; thus we have

$$
R=R_{2 b}-R_{2 b}^{\infty}+M^{-1} \text {. }
$$

The term $R_{2 b}^{\infty}$ is subtracted from $R_{2 b}$ since this term is already included in $M^{-1}$.

The average drag coefficient of an aggregate is extracted from the relation between the total force exerted on the particles if each particle were translating at the same velocity, without rotation, and in the absence of a shearing motion $E^{\infty}=0$ :

$$
F=\left(\sum_{\alpha=1}^{N} \sum_{\beta=1}^{N} R_{F U}^{\alpha \beta}\right) U
$$

The average drag coefficient corresponding to an average of the force for any orientation of the aggregate relatively to the velocity will be given by the trace of the resistance matrix coupling the force and the velocity

$$
\xi=\sum_{\alpha=1}^{N} \sum_{\beta=1}^{N} T_{r} R_{F U}^{\alpha \beta}
$$

The stress on the particles is obtained from the general relation (1). For freely suspended particles, the forcetorque vector $F$ is equal to zero and we have

$$
S=\left[R_{S U}(R)^{-1}{ }_{U F} R_{F E}-R_{S E}\right]: E^{\infty}=R_{S E}^{t}: E^{\infty} .
$$

In the absence of symmetry, the tensor $S_{i j}^{\alpha}$ would be related to the velocity gradient tensor on the particle $\beta$ by

$$
S_{i j}^{\alpha}=T_{i j k l}^{\alpha \beta} E_{k l}^{\beta} \text {. }
$$

If we rolate the aggregate relative to the shear flow in order to average the stress over all the orientations, we get $^{7}$

$$
\begin{aligned}
\left\langle S_{m n}^{\alpha}\right\rangle= & \left(-\frac{1}{15} \delta_{i j} \delta_{k l}+\frac{1}{10} \delta_{i l} \delta_{j k}+\frac{1}{10} \delta_{i k} \delta_{j l}\right) \\
& \times T_{i j k l}^{\alpha \beta} E_{m n}^{\beta},
\end{aligned}
$$

where $\delta_{i j}$ is the Kronecker delta. As the two tensors $S$ and $E$ have only five independent components, we can replace the four indices $i, j, k, l=1,3$ of $T$ by two $i, j=1,5$, and from Eqs. (5) and (7), we have

$$
\left\langle S_{23}\right\rangle=\sum_{\alpha} \sum_{\beta}\left\langle\left(R_{S E}^{t}\right)^{\alpha \beta}\right\rangle E_{23}^{\infty},
$$

with

$$
\begin{aligned}
\left\langle\left(R_{S E}^{t}\right)^{\alpha \beta}\right\rangle= & \frac{4}{1 D}\left[R_{11}+R_{22}+R_{33}\right. \\
& \left.+R_{44}+R_{5 S}+\frac{1}{2}\left(R_{15}+R_{51}\right)\right] .
\end{aligned}
$$

Here $R$ stands for the $5 \times 5$ matrix $\left(R_{S E}^{t}\right)^{\alpha \beta}$ and $E_{23}^{\infty}=\gamma / 2$, where $\gamma$ is the shear rate.

We have checked our numerical procedure against Batchelor and Green's results for two spheres ${ }^{8}$

$$
\begin{aligned}
T_{i j k l}= & \frac{1}{2}\left(\delta_{i k} \delta_{j l}+\delta_{i l} \delta_{j k}\right)(1+K) \\
& +\left(e_{i} e_{k} \delta_{j l}+e_{j} e_{k} \delta_{i l}-e_{k} e_{l \frac{2}{3}} \delta_{i j}\right) L \\
& +e_{k} e_{l}\left(e_{i} e_{j}-\frac{1}{3} \delta_{i j}\right) M,
\end{aligned}
$$

where $K, L$, and $M$ are tabulated functions of the separation between the spheres and $e$ is a unit vector along the line of center. From Eq. (7), we get

$$
\left\langle S_{23}\right\rangle=\left(1+K+\frac{2 L}{3}+\frac{2 M}{15}\right) E_{23}^{\infty}=(1+J) E_{23}^{\infty} .
$$

The value of $J$ (which depends on the separation between the spheres) gives the contribution to the stress from the hydrodynamic interaction between two spheres; the one is the stress on two isolated spheres for a shear rate unity and gives Einstein's viscosity for a dilute suspension of noninteracting spheres. For two spheres at contact $J \approx 0.22$. We have verified that we recover this result and also that the average value $\left\langle S_{23}\right\rangle$ is invariant when we change the orientation of the aggregate.

\section{RESULTS FOR FRACTAL AGGREGATES}

We built random aggregates by a hierarchical model of cluster-cluster aggregation. ${ }^{9}$ We begin with the total number of particles $N$ we want in the aggregate, then we form $N / N I$ aggregates of $N I$ particles and form the second generation by sticking them together after a random rotation and a random ballistic trajectory of each cluster. The same procedure is used at each generation and we end up with the aggregate of $N$ particles. The fractal behavior is verified in Fig. 1, where we get a straight line by plotting the logarithm of the radius of gyration vs the number of particles. The slope gives a fractal dimension $d_{f} \approx 2.00$ which is closed from the value 2.03 usually found with this model. ${ }^{9}$

The mobility matrix $M$ contains $(11 N)^{2}$ elements, corresponding to 11 degrees of freedom per particle. To determine the hydrodynamic properties of an aggregate we need to average over different, randomly generated aggregates, typically between 5 and 10 , and thus we are limited to a few hundred particles. (The maximum number used here is $N=243$ ).

The drag coefficient is defined by Eq. (4). However, instead of using the total resistance matrix, we have only used the mobility invert in computing the drag. We have done this because the lubrication part which gives a divergent force if two particles have a finite relative velocity is not important when all the particles move at the same velocity. ${ }^{10}$ This can be understood from the fact that $\left(R_{2 b}-R_{2 \infty}\right) U^{\infty}$ 


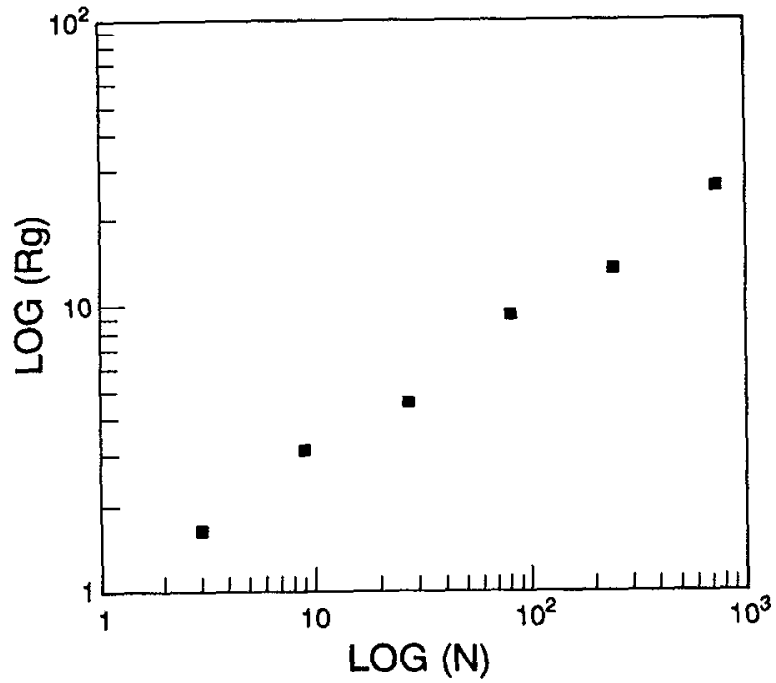

FIG. 1. The fractal dimension of the aggregate built by cluster-cluster aggregation is given by the slope of the logarithm of the radius of gyration as a function of the number of particles; we find $d_{f}=2.00$

corresponds to the forces owing to multipoles higher than two for two particles alone in the fluid whose velocity far from the particles is $U^{\infty}$. Inside an aggregate, the velocity field is screened and adding the pairwise term $\left(R_{2 b}-R_{2 \infty}\right)$ $U^{\infty}$ misrepresents this screening resulting in an error that can become significant for large, compact aggregates. For our aggregates of fractal dimension 2, we obtain a drag which is $7 \%$ larger by including $R_{2 b}-R_{2 \infty}$ for the smaller aggregates ( $N=27$ particles) and $20 \%$ larger for large aggregates ( $N=243$ particles). For a compact spherical cluster formed by hexagonally packing 209 spheres resulting in a radius of $6.54 a$ ( $a$ is the radius of the sphere and the value of 6.54 corresponds to the distance from the center of the clus-

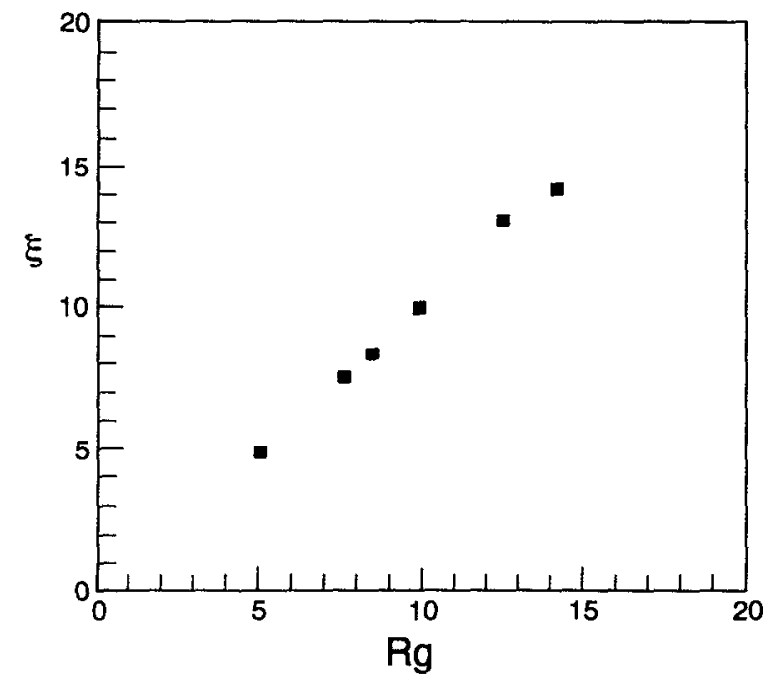

FIG. 2. The drag coefficient obtained from the invert of the mobility matrix as a function of the radius of gyration. ter to the center of the most distant sphere), the drag from the mobility invert $\xi_{m}=6.75$ is in fair agreement with Stokes law; adding lubrication, however, gives $\xi=17.9$ almost three times the correct value.

In Fig. 2, we have plotted the drag coefficient $\xi_{m}$ as a function of the radius of gyration. The relationship is lincar; within the uncertainty, the slope is equal to one. Thus we confirm previous results and the standard thinking ${ }^{1-13}$ that identifies the hydrodynamic radius with the radius of gyration. Note, from the definition of the drag all particles move with the same velocity as would be the case for a fixed aggregate. With the same reasoning, we should expect that the stress on a cluster, or said differently, the scaling of the viscosity with size should be proportional to the cube of the radius of gyration; i.e., $S \approx\left(R_{g} / a\right)^{3} \gamma / 2$.

In Fig. 3, we have plotted the stress as a function of $R_{g}^{3}$; it is obvious that $S$ does not scale linearly with $R_{g}^{3}$. if instead we plot the stress as a function of the number $N$ of particles making up the aggregate, as done in Fig. 4, we have a linear relationship with a slope slightly less than one. Thus the stress scales linearly with the number of particles for a free aggregate, i.e., one in which the particles can move freely because there is no force or torque exerted on them [cf. Eq. (5) ], rather than being proportional to the cube of the largest dimension as one would expect from low-Reynold-number hydrodynamics for a rigid object. We shall show below in the discussion, however, that for a fixed aggregate-one in which the particles are constrained to have no relative motion-the stress does indeed scale as the cube of the largest dimension or as $N^{3 / d}$.

One should note that lubrication has been included in the calculation of the stress. In a shear flow, particles do not all move at the same velocity and lubrication is very important in slowing the relative motion of particles and preventing them from coming into contact. As a result, a large portion of the stress is associated with the lubrication forces and the particle relative velocities. For a fractal aggregate, the

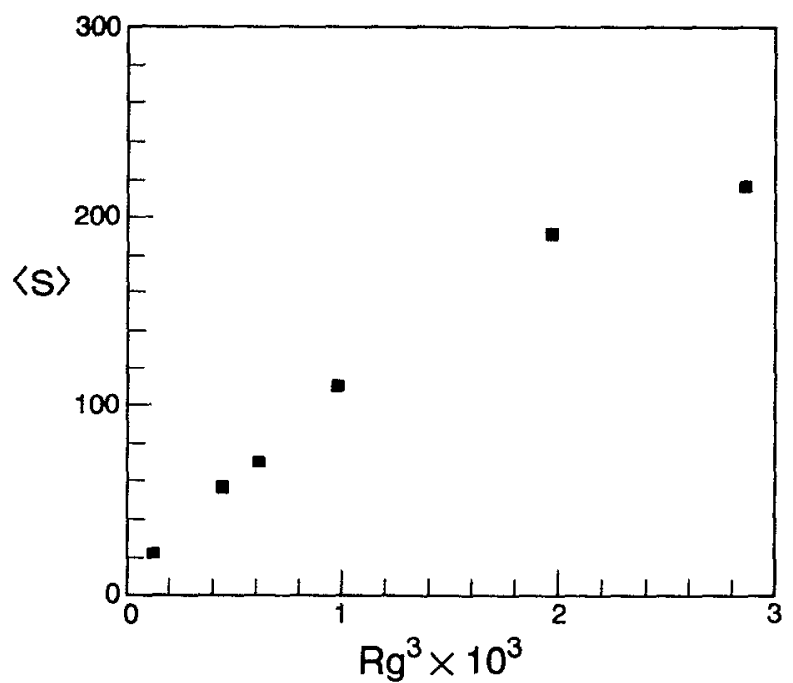

FIG. 3. Total average stress (for a shear rate $\gamma=1$ ) as a function of the cube of the radius of gyration. 


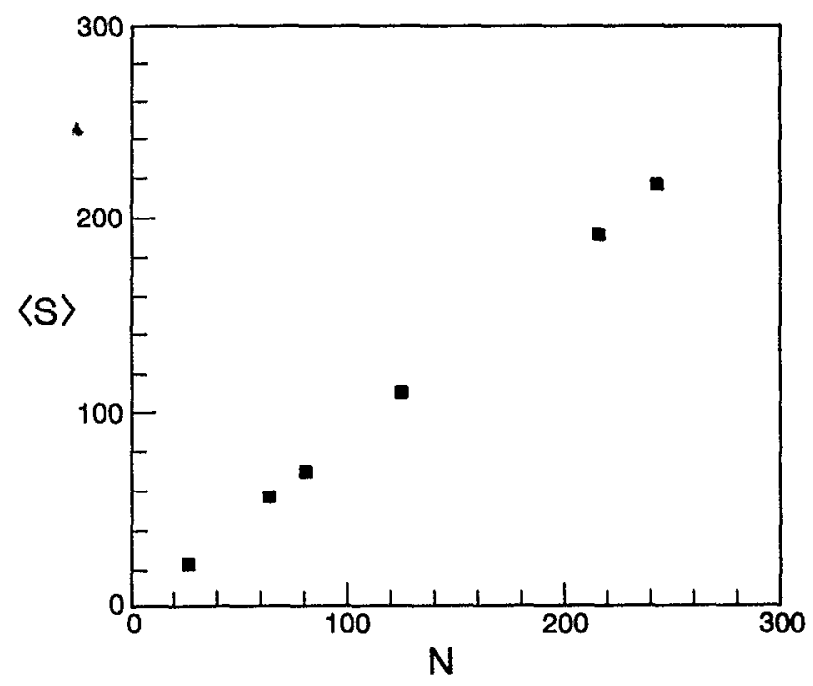

FIG. 4. Total average stress as a function of the number of particles in the aggregate.

stress coming from the first two multipoles, as expressed by $M$, represents only $40 \%$ of the total stress. In a compact spherical cluster of 209 spheres, the first two multipoles give only $10 \%$ of the total stress.

\section{DISCUSSION}

In order to understand the behavior of the stress on a fractal aggregate and the important difference between free and fixed aggregates, we can examine the simpler special case of a straight line of spheres, where the average stress is known to grow as $l^{3} / \log 2 l$, where $l=$ is the half-length of the chain normalized by the radius of the spheres.,14,15

In this degenerate case of a linear chain, or a regular fractal (see below), the stress grows as $N^{3}$ whether the particles are free or fixed. For fixed particles, this scaling is obvious, but for free particles, it requires an analysis of the relative velocity of the particles in the chain and the stress due to this relative velocity coming from the lubrication forces. For the free chain, the $N^{3}$ law can be recovered as follows:

Because of the linearity of Stokes flow, we can determine the stress by first supposing the particles to be fixed, calculate the hydrodynamic and interparticle density force required to keep them fixed, and then set the force to zero to determine the relative velocities. The relative velocities of near touching particles will then give the dominant contribution to the stress from $R_{S U}$ in Eq. (1).

The hydrodynamic force arising from an external flow on a particle $k$ is proportional to the velocity at its center. If the axis of the chain lies in the shear plane with an orientation characterized by the unit vector $e$, we have for the force on the particle $k$ along the axis of the chain

$$
f_{r}^{(k)}=C e_{1} e_{2} l_{k} \text {, where } l_{k}=(2+\epsilon) k \text {, }
$$

The separation between two spheres in the chain normalized by the radius is $2+\epsilon$, with $\epsilon \ll 1$. The constant $C$ is a screen- ing factor for a single sphere alone $C=1$. If we add by pairs the screening of the two neighbors, we get

$$
C=1+2\left(X_{11}^{A}-1+X_{12}^{A}\right)=0.29,
$$

where $X_{11}^{A}$ is the appropriate component of the resistance matrix.

The total screening cannot be obtained without taking into account the many-body problem through the inversion of the mobility matrix. This long-range screening is responsible for the logarithmic term, in the scaling of the shear, which we shall neglect in the following derivation of the stress.

If we consider a pair of spheres, their relative radial velocity will be given by

$$
V_{r}^{i}-V_{r}^{i-1}=2 \epsilon\left(F_{r}^{i}-F_{r}^{i-1}\right),
$$

where $\epsilon$ is the separation between the surfaces of the spheres $i$ and $i-1$ and $F_{r}^{i}$ (respectively, $F_{r}^{i-1}$ ) is the total force acting on the sphere $i$ (respectively, $i-1$ ). The total force is the sum of the hydrodynamic force $f_{i}$ and the tension exerted by a neighboring sphere

$$
F_{i}=f_{i}+T_{i},
$$

where $T_{i}$ is the tension exerted by the sphere $i+1$ on the sphere $i$. Also, we have $T^{i}=f^{i+1}+T^{i+1}$ and finally

$$
\begin{aligned}
& F^{i}=\sum_{k=i}^{N} f_{r}^{(k)}, \\
& F^{i-1}=\sum_{k=-N}^{i-1} f_{r}^{(k)}=\sum_{k=-N}^{-i} f_{r}^{(k)}=-\sum_{k=i}^{N} f_{r}^{(k)},
\end{aligned}
$$

where we have used the symmetry of the radial forces

$$
f_{r}^{(k)}=-f_{r}^{(-k)} \text {. }
$$

The force coming from the shear $F_{c i s}=R_{F E}: E$ cancels by symmetry if we consider the effect on one sphere of its two neighbors. This is not true for the last spheres in the chain where we get

$$
f_{r}^{(N)}=\gamma e_{1} e_{2}\left(0.645 l_{N}+1.392\right) .
$$

The relative force is then given by

$$
F_{r}^{(i)}-F_{r}^{(i-1)}=2\left(\sum_{k=i}^{N-1} f_{r}^{(k)}+f_{r}^{N}\right) .
$$

Putting together the relations (11) (with $C=0.29$ ), (12), and (15), we have

$$
\begin{aligned}
V_{r}^{(i)}-V_{r}^{(i-1)}= & \epsilon \gamma e_{1} e_{2} \\
& \times\left[1.16\left(N^{2}-i^{2}+i\right)+4 N+11.1\right]
\end{aligned}
$$

Now, the stress on the particles can be split into a part coming from the relative velocities and another part coming from the configuration. If the chain is oriented along the dilatation or the compression axis, the relative radial velocities grow as $N^{2}-i^{2}$, and this will be the principal contribution to the stress. The stress on a particle due to the relative velocity in the lubrication regime $\epsilon \ll 1$ scales as $(3 / 4 \epsilon) \Delta V$, hence

$$
S_{12}\left(\theta=45^{\circ}\right) \approx 0.6 \sum_{i=1}^{N} \frac{3}{4 \epsilon}\left(V_{r}^{(i)}-V_{r}^{(i-1)}\right),
$$




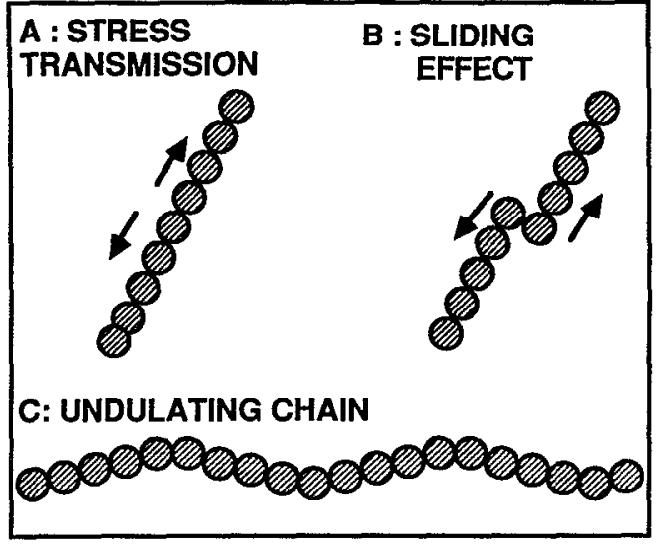

FIG. 5. (A) Transmission of the stress in a shear flow; the stress is maximum on the central sphere; (B) sliding effect; the total stress is half of case (A) and is maximum at the center of the two segments; (C) undulated chain.

or with Eq. (17) for a shear rate equal to one

$$
S_{12}\left(\theta=45^{\circ}\right)=1.044 N^{3}+3.83 N^{2}-1.044 N \text {. }
$$

This result shows that the $N^{3}$ behavior arises from the transmission of tensions from one sphere to the other by the lubrication forces. If we break the alignment on the compression axis, as shown in Fig. 5, however, we allow the spheres to slip tangentially and the tension is no longer transmitted from one part of the chain to the other. In the case of the straight line [Fig. 5(A)], we have a total stress for 81 particles $S_{23}=2473$, whereas in the other case [Fig. 5(B)], where the chain has been broken in two segments, but with the same separation $\epsilon=10^{-5}$ between the spheres, we get $S_{23}=1194$. The stress has been halved and the distribution of stresses is very different. In the first case, the stress is maximum at the center of the chain and in the second case it is maximum at the center of the two half-chains.

A simple estimate of the change in viscosity brought about by cutting a chain in half goes as follows: for a linear chain of $N$ particles, the stress grows as $N^{3}$. Cutting this chain in half, we have two chains each with $N / 2$ particles. Thus the stress goes as $2(N / 2)^{3} \approx 1 / 4 N^{3}$. That the numerical calculations give $1 / 2$ rather than $1 / 4$ results from the fact that the two half-chains interact strongly hydrodynamically, increasing the viscosity. Note that if the particles were rigidly connected, then the break in the middle as shown in Fig. 5 would have a negligible effect on the stress which would still grow as $L^{3}$.

The perfectly linear chain of almost touching particles is a special and degenerate case in that the free aggregate scales the same as the fixed, i.e., as $N^{3}$ rather than $N$. On the other hand, a spherical compact aggregate with $d_{f}=3$ is also degenerate in that the stress scales as $L^{3}=N$; thus both fixed and free agree as to the stress scaling.

Rather than a single defect relative to a straight line as discussed above, we can take an undulating or snakelike chain. We have chosen an undulated chain [cf. Fig. 5(c)] with a period of 18 spheres and an amplitude of 2.68 radii. Here too, we get a stress which is very small compared to a straight line of the same length or end-to-end distance. For instance, for a straight line of 75 spheres, $S_{23}=2019$, whereas for the undulated chain, we have $S_{23}=293$. Furthermore, if we plot the stress as a function of the number of spheres for this undulated chain, we recover a linear dependence for a large enough $N$, just as in the fractal case. It is clear then that depending on the structure of the aggregate, there exists a characteristic length $L_{\mathrm{c}}$ for which the stress is transmitted as in a rigid body for $L<L_{c}$, and when $L>L_{c}$, we find the linear dependence of the stress as a function of the number of particles. For random fractal aggregates, this characteristic length is certainly of the same order of magnitude as the distance between two spheres since they stick together randomly. In contrast, for a rcgular fractal aggregatc, there are some straight lines which join the extremities of the aggregate, and we expect that the characteristic length is just the whole size of the aggregate. For example, for a regular fractal of 125 particles and a radius of gyration of 17, we get $\left\langle S_{23}\right\rangle=410$ instead of $\left\langle S_{23}\right\rangle=100$ for the same radius of gyration with a random aggregate. For a curved chain of spheres, one might think that the persistance length could play the role of this characteristic length for the transmission of stress.

The persistance length $L_{p}$ characterizes the decrease in the correlations between the orientations of different segments of the chain

$$
\langle\mathbf{e}(0) \mathbf{e}(l)\rangle \propto e^{-l / L_{P}} .
$$

Here, $\mathrm{e}$ is a unit vector joining two contiguous particles and $l$ is the distance separating the two vectors along the chain.

We constructed some freely rotating polymer chains. The vector $\mathbf{r}_{n}$ joining the sphere $n-1$ to the sphere $n$ makes a constant polar angle $\theta$ with the vector $\mathbf{r}_{n-1}$, but the azimuthal angle is chosen randomly. For this polymer model, the persistance length is given by ${ }^{16}$

$$
\frac{L_{p}}{a}=\frac{2}{1-\cos \theta}
$$

For a given number of particles in the chain, we expect, of course, that the total average stress increases as $L_{p}$ increases. This is what we observe in Fig. 6 where we have plotted the average stress as a function of $L_{p}$ for a chain of 131 particles. If we model the chain as an ensemble of rigid rods of length $L_{p}$, we could expect that the total stress will increase as

$$
\langle S\rangle \propto \frac{N}{L_{p}} L_{p}^{3} \propto N L_{p}^{2} .
$$

Actually, for a given $N$, we find a linear law as a function of $L_{p}$ (cf. Fig. 6). We can check the hypothesis that for a length $L<L_{p}$, the stress grows as $N^{3}$. For a persistance length of 65 particles, we have drawn in Fig. 7 a log-log plot of the stress as a function of the number of particles. We find that for $N<65$ we get a power law: $S \propto N^{1.8}$ instead of $S \propto N^{3}$. This means that only a small amount of disalignment which always persists for $L<L_{p}$ is enough to lower the transmission of the stress and the exponent of the power law. Instead of Eq. (20), we have now 


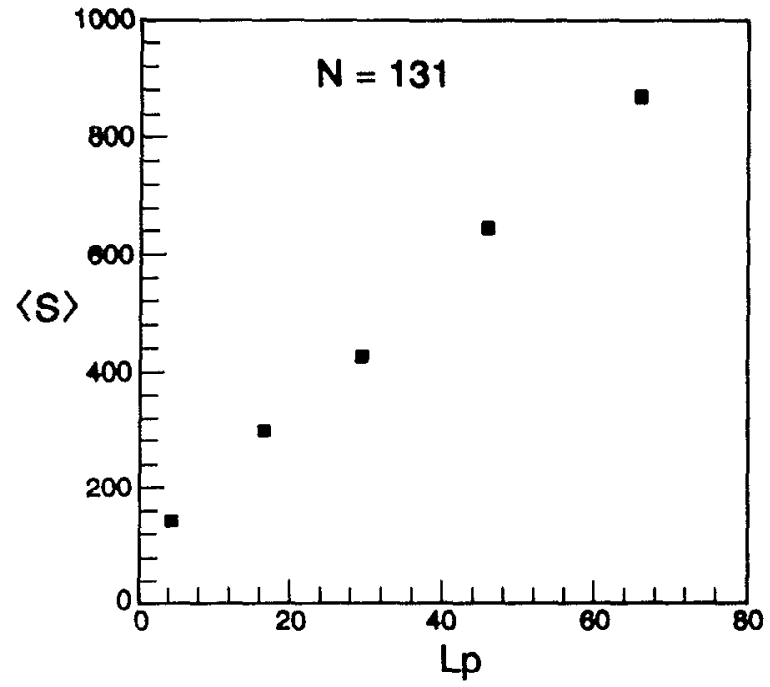

FIG. 6. Total averaged stress as a function of the persistance length for a chain containing 131 particles.

$$
\langle S\rangle \propto \frac{N}{L_{p}} L_{p}^{1.8} \propto N L_{p}^{0.8}
$$

which is not far from the linear behavior that we observe.

We have supposed that the polymer was composed of independent rods of length $L_{p}$, but in a real chain, where all these rods are connected, the stress will be larger, which might explain the difference between the law in $L_{p}^{0.8}$ from this simple model and the linearity in $L_{p}$ obtained from the numerical simulation. Another point is that in writing Eq. (20) or Eq. (21), we ignore the hydrodynamic screening of the velocity field inside the cluster, or in other words we consider the free draining limit. For porous aggregates, Johnson and Segalman ${ }^{1}$ have introduced the impermeability

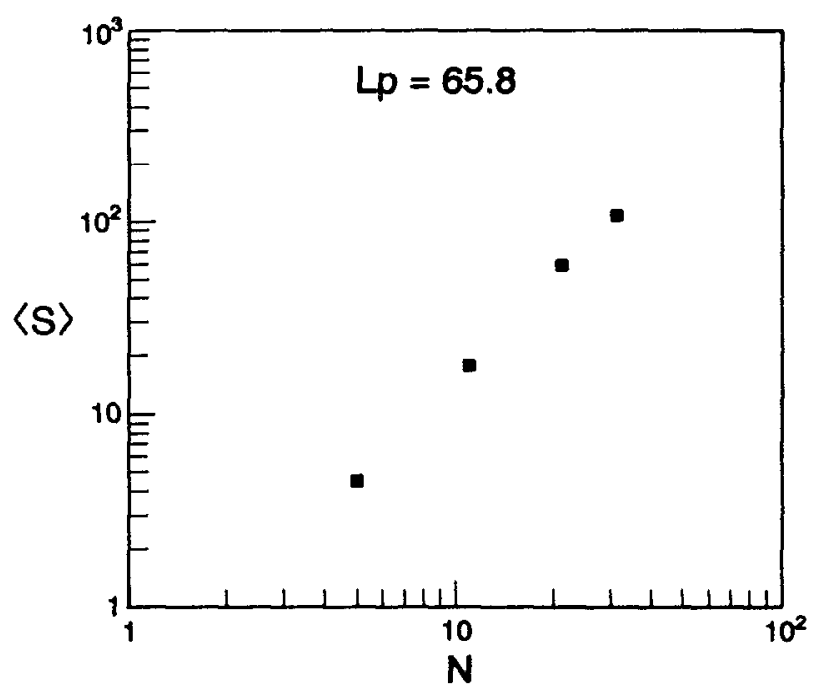

FIG. 7. The log-log plot of the average stress as a function of the number of spheres for a persistance length of 65 particles (corresponding to a polar angle of $10^{\circ}$ between each particle).

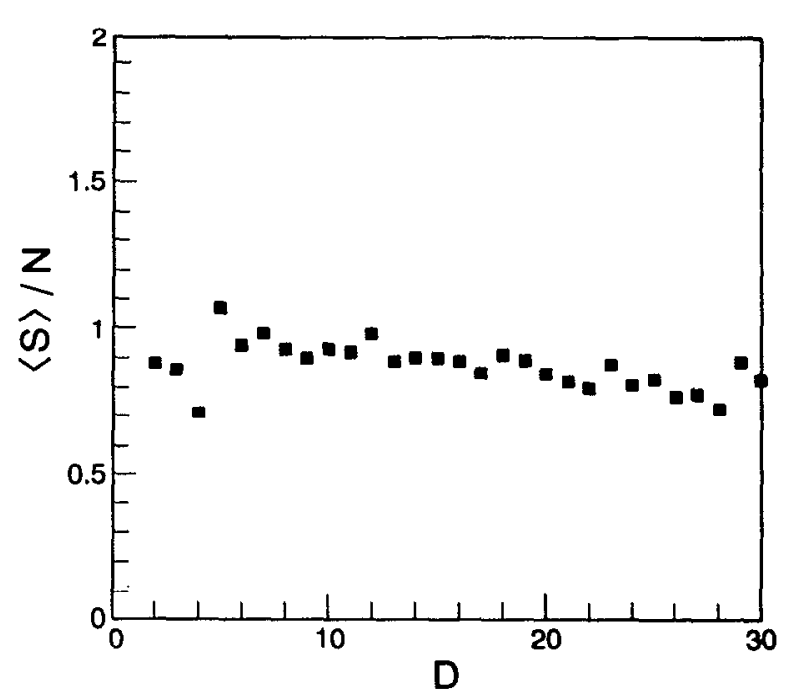

FIG. 8. Average stress by particle as a function of the distance from the center of the aggregate for a random aggregate of 243 particles.

parameter $\gamma^{2}=2 N / R_{H}$ (where $R_{H}$ is the hydrodynamic radius normalized by the radius of a sphere) to study the modification of the flow by a porous aggregate. For our simulations, this parameter is in the range $10<\gamma^{2}<30$, where the effect of the long-range hydrodynamic screening is still weak.

As another indication that the screening of the shear inside the aggregate is not important, we can look at the distribution of stress inside the aggregate. In Fig. 8, we have plotted the average stress on a particle as a function of its distance from the center of the aggregate; this distribution is rather flat and does not indicate a screening of the field. We can also note that for a regular fractal where the persistance length of the chain is equal to the external diameter, the stress increases from the extremity to the center parabolically just as in the case of a rigid rod.

From this study, we can tentatively try to deduce the hydrodynamic viscosity of an aggregated colloidal suspension. This hydrodynamic viscosity can be measured with an oscillatory shear at high enough frequencies so that its structure does not have the time to deform ${ }^{17}$ as explained in the Introduction. We have

$$
2 \mu_{\mathrm{eff}} \gamma=2 \mu \gamma+\frac{N}{V}\left\langle\frac{S^{H}}{N}\right\rangle
$$

where $S^{H} / N$ is the total hydrodynamic stress per particle. If we assume that $S^{H}=C N L_{\rho}$, we get a simple Einstein law

$$
\frac{\mu_{\mathrm{eff}}}{\mu}=1+\frac{5}{2} C L_{p} \varphi
$$

with a constant different from 2.5. The scaling of $C$ and $L_{p}$ with $\varphi$ and the shear rate now determine the rheological response.

We have shown here that the scaling of the hydrody- 
namic stress on an aggregate with the number of particles generally falls into two classes. For a free random aggregate-force- and torque-free particles held together by hydrodynamic forces, which is also the high frequency behavior of flexible objects, - the stress scales linearly with the number of particles. For a fixed aggregate-particles held rigidly together which is also the low frequency behavior of flexible objects-the stress scales as the cube of the largest length, $L^{3}=N^{3 / d_{f}}$, where $d_{f}$ is the fractal dimension. The case of a regular fractal, such as a strict linear chain with $d_{f}=1$, always behaves as a fixed aggregate, independent of the form of the interparticle connections.

\section{ACKNOWLEDGMENTS}

The work was supported by NATO and CNRS/NSF grants. Computer time has been provided by the Centre de Calcul vectoriel pour la Recherche.
'M. W. Johnson and D. J. Segalman, J. Non-Newtonian Fluid Mech. 9, 33 (1981).

${ }^{2}$ P. F. Mijnlieff and F. N. Wiegel, J. Polymer Sci. 16, 245 (1978).

${ }^{3}$ J. F. Brady and G. Bossis, J. Fluid Mech. 155, 105 (1985).

${ }^{4}$ G. Bossis and J. F. Brady, J. Chem. Phys. 91, 1866 (1989).

${ }^{5} \mathrm{~J}$. F. Brady and G. Bossis, Annu. Rev. Fluid Mech. 20, 111 (1988).

${ }^{6} \mathrm{G}$. Bossis and J. F. Brady, in Hydrodynamics of Dispersed Media, edited by J. P. Hulin (Elsevier, Amsterdam, 1990).

${ }^{7}$ R. B. Bird, O. Hassager, R. C. Armstrong and C. F. Curtiss, Dynamics of Polymeric Liquids (Wiley, New York, 1976), p. 625.

${ }^{8}$ G. K. Batchelor and J. T. Green, J. Fluid Mech. 56, 401 (1972).

${ }^{9}$ R. Jullien and R. Botet. Aggregation and Fractal Aggregates (World Scientific, Singapore, 1987).

10 R. J. Phililips, J. F. Brady, and G. Bossis, Phys. Fluids 31, 3462 (1988).

"J. G. Kirkwood and J. Riseman, J. Chem. Phys. 16, 565 (1948).

${ }^{12}$ Z. Y. Chen, J. M. Deutch, and P. Meakin, J. Chem. Phys. 80, 2982 (1984).

${ }^{13}$ M. Doi and S. F. Edwards, The Theory of Polymer Dynamics (Oxford University, Oxford, 1986).

${ }^{14}$ G. K. Batchelor, J. Fluid Mech. 44, 419 (1970).

is J. G. Kirkwood and P. L. Auer, J. Chem. Phys. 19, 281 (1951).

${ }^{10}$ P. J. Flory, Statistical Mechanics of Chain Molecules (Wiley, New York, 1969).

17 J. C. Van der Werff and C. G. de Kruif, Phys. Rev. A 39, 795 (1989). 